\title{
Laparoscopic stapler repair of high rectovaginal fistula: A case report
}

\author{
Amit Kumar Parmar, Mittu John Mathew, Prasanna Kumar Reddy* \\ Department of Surgical Gastroenterology and Minimal Access Surgery, Apollo Hospital, Chennai, India \\ Email: *drpkreddyapollo@gmail.com
}

Received 3 October 2012; revised 3 November 2012; accepted 11 November 2012

\begin{abstract}
For thousands of years, women simply tolerated the distressing symptoms generated by rectovaginal fistulas (RVFs). This is no longer necessary because most RVFs can be surgically corrected via a number of approaches. Most rectovaginal fistulas are acquired; obstetric injury alone accounts for nearly $88 \%$ of the cases. The high fistulas are repaired by abdominal approach, while middle or low fistulas are best approached perineally. There are only few case reports of laparoscopic RVF repair noted in literature till date. Laparoscopic repair of RVF is challenging and requires advanced laparoscopic skill. Laparoscopy is a better alternative in selected cases of RVF and yields faster recovery and good patient compliance. We present a case of high RVF managed laparoscopically by using stapler.
\end{abstract}

Keywords: Laparoscopy; Rectovaginal Fistula; Stapler

\section{INTRODUCTION}

Obstetric trauma is the most common cause of rectovaginal fistula. Most of the RVFs are managed by conventional open surgery. Abdominal surgeries such as hysterectomies, low anterior resections and ileo-anal anastomosis also carry the risk of developing an RVF. RVF can also develop secondary to radiation, pelvic malignancy, and diverticular disease. Low RVF can be repaired through perineal approach but high RVF requires transabdominal approach. Laparoscopic approach is feasible in high RVF.

\section{CASE PRESENTATION}

A 36-year-old female patient underwent total abdominal hysterectomy with bilateral salpingoophorectomy for endometriosis 6 months back elsewhere. On postoperative day 3 , she had severe abdominal pain and distension

\footnotetext{
"Corresponding author.
}

for which she was re-explored and found to have a rectal perforation. Primary closure of perforation and diverting ileostomy was performed. Post operatively she developed high RVF which did not healed even after 3 months of conservative management. She was referred to our hospital for further management. After doing all routine investigations and methylene blue leak test, she was posted for laparoscopic repair of RVF.

\section{OPERATIVE TECHNIQUE}

Under general anaesthesia in modified lithotomy position, pneumoperitoneun was created by veress needle through Palmer's point. $10 \mathrm{~mm}$ supraumblical telescopic port was placed. $5 \mathrm{~mm}$ and $12 \mathrm{~mm}$ ports inserted in left and right iliac fossae respectively. Adhesiolysis was done with sharp dissection. The rectum was mobilised. A short, wide fistulous tract was identified Figure 1 and dissected all around and divided with articulating stapler (Echelon flex 60, ETHICON ${ }^{\mathrm{TM}}$ ). Air leak test was performed which showed no leak. The omentum was placed between rectum and vagina. Ileostomy was closed. The patient was discharged on 3rd post operative day. She is symptom free on 6 months follow up.

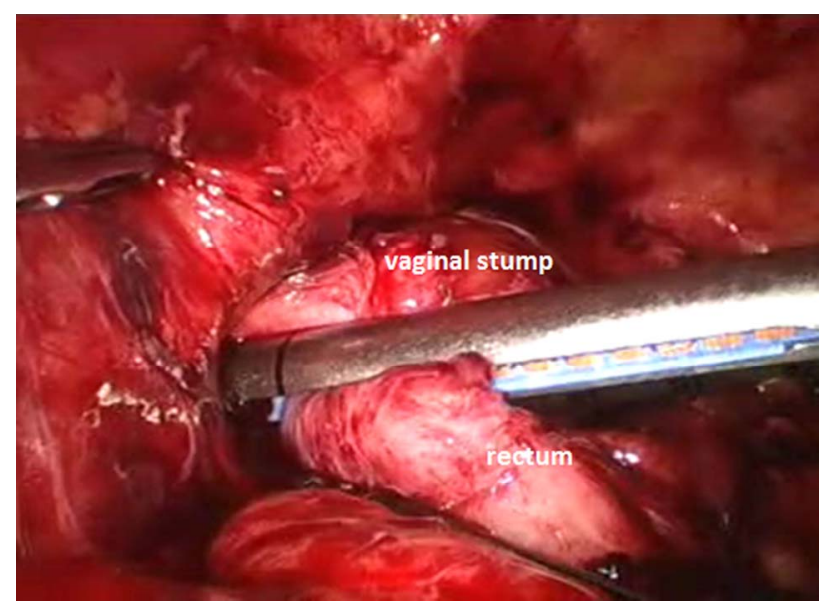

Figure 1. Intraoperative view of division of rectovaginal fistula with articulating stapler. 


\section{DISCUSSION}

Rectovaginal fistula is epithelial lined communication between rectum and vagina. Most common cause of RVF is obstetric trauma. It can occur as a complication after variety of rectal, vaginal and pelvic operations including hysterectomy, low anterior resection, ileal-pouch anal anastomosis and stapled hemorroidectomy [1]. RVF can be associated with pelvic malignancy or radiation therapy for malignancy, and inflammatory bowel disease. RVFs can be classified into low and high varieties. Low RVF is between the lower third of the rectum and the lower half of the vagina. A high RVF is between the middle third of the rectum and the posterior vaginal fornix. Small-sized fistulas are less than $0.5 \mathrm{~cm}$ in diameter, medium-sized fistulas are $0.5-2.5 \mathrm{~cm}$, and large-sized fistulas exceed $2.5 \mathrm{~cm}$ [2]. Clinical features of the rectovaginal fistula are stool and air passage from the vagina. Other symptoms include recurrent urinary tract infection and perineal skin inflammation or infection. Symptoms of chronic inflammation and irritation in these patients have an effect on their social life and psychology and lead to sexual dysfunction. Most fistulas at the lower rectum are palpable by digital rectal examination. Contrast radiography is the most dependable method for diagnosing small and high rectovaginal fistulas. High fistulas may not be readily apparent on physical examination or vaginal inspection and may even be missed by endoscopy. Methylene blue enema with a vaginal tampon in place, looking for staining on the tampoon is used to confirm the diagnosis. Vaginography with a water soluble contrast medium has a reported sensitivity of $79 \%$ to $100 \%$ [3-5]. CT and MRI also play a role in the diagnosis and evaluation of the RVF as they may give insight into the underlying cause of the fistula.

Spontaneous healing may occur with adequate medical treatment such as total parenteral nutrition, antibiotics, and long-time fasting. However, surgical therapy remains the mainstay for managing complex fistula was not suitable for conservative management or underwent prolonged conservative management (without resolution). Operative access to this type of lesion includes fecal diversion, and/or transperineal approach of resection, or rectal anastomosis or repair. Operation of the middle and lower rectum is associated with complications, including urinary and sexual dysfunction. The management of RVF depends on size, location, cause, anal sphincter function and overall health status of the patient. Low fistulas can be repaired through perineal approach. Transabdominal approach is standard for high fistula. Total laparoscopic repair of RVF is still rare. Nezhat $\mathrm{CH}$ et al. [6] reports correction of two cases of RVF by laparoscopy. Pelosi et al. [1] reported laparoscopic upper rectovaginal mobilisation with transvaginal repair of recurrent RVF. Pala- nivelu et al. [2] reported 2 cases of high RVF managed laparoscopically. Schwenk et al. [7] reported a case of intracorporeal colorectal anastomosis for which they had performed a laparoscopic resection of the sigmoid colon with the fistulous tract and. They all concluded that laparoscopic repair of RVF is feasible but it demands adequate experience in advanced laparoscopic procedures and proper identification of tissue planes. Good preparation of the bowel is essential to avoid any faecal contamination of the operative area. Fistulous tract is generally divided and closed by using suture. But, in our case we used linear stapler for this purpose. The idea behind using it was to make procedure simpler and faster and avoidance of faecal contamination as well. And thus, we were able to close the ileostomy at the same time and avoided one more surgical burden on the patient. This is probably first case of laparoscopic stapler repair of RVF in literature.

\section{CONCLUSION}

Laparoscopic repair of RVF is challenging and requires advanced laparoscopic skill. Laparoscopy is a promising alternative in selected cases of RVF and yields faster recovery and good patient compliance. We found that stapler repair as compared to primary intracorporeal closure makes the procedure simpler, faster, and easy. Safety and long term outcomes of laparoscopic repair is yet to be proved by long term follow up and further studies.

\section{REFERENCES}

[1] Pelosi III, M.A. and Pelosi, M.A. (1997) Transvaginal repair of recurrent rectovaginal fistula with laparoscopicassisted rectovaginal mobilization. Journal of Laparoendoscopic \& Advanced Surgical Techniques, 7, 379-383. doi:10.1089/lap.1997.7.379

[2] Palanivelu, C., Rangarajan, M., Senthilkumar, R., Madankumar, M.V. and Kalyanakumari, V. (2007) Laparoscopic management of iatrogenic high rectovaginal fistulas (type VI). Singapore Medical Journal, 48, e96-e98.

[3] Arnold, M.W., Aguilar, P.S. and Stewart, W.R.C. (1990) Vaginography: An easy and safe technique for diagnosis of colovaginal fistulas. Diseases of the Colon \& Rectum, 33, 344-345. doi:10.1007/BF02055482

[4] Bird, D., Taylor, D. and Lee, P. (1993) Vaginography: The investigation of choice for vaginal fistulae? Australian and New Zealand Journal of Surgery, 63, 894-896. doi:10.1111/j.1445-2197.1993.tb00366.x

[5] Giordano, P., Drew, P.J. and Taylor, D. (1996) Vaginography-Investigation of choice for clinically suspected vaginal fistulas. Diseases of the Colon \& Rectum, 39, 568-572. doi:10.1007/BF02058713

[6] Nezhat, C.H., Bastidas, J.A., Pennington, E., Nezhat, F.R., Raga, F. and Nezhat, C.R. (1998) Laparoscopic treatment 
of type IV rectovaginal fistula. Journal of the American Association of Gynecologic Laparoscopists, 5, 297-299. doi:10.1016/S1074-3804(98)80036-4

[7] Schwenk, W., Bohm, B., Grundel, K. and Muller, J.
(1997) Laparoscopic resection of high rectovaginal fistula with intracorporeal colorectal anastomosis and omentoplasty. Surgical Endoscopy, 11, 147-149. $\underline{\text { doi: } 10.1007 / \mathrm{s} 004649900318}$ 